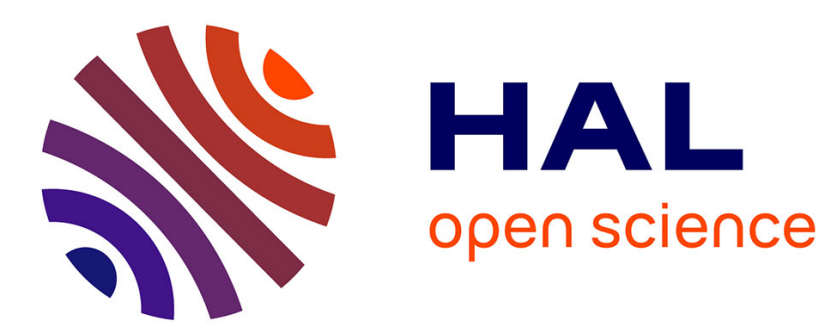

\title{
Video Stream Transmodality
}

Pierre-Olivier Rocher, Christophe Gravier, Julien Subercaze, Marius Preda

\section{To cite this version:}

Pierre-Olivier Rocher, Christophe Gravier, Julien Subercaze, Marius Preda. Video Stream Transmodality. International Conference on Enterprise Information Systems, Apr 2014, Lisboa, France. pp.28, 10.5220/0004892000280037 . hal-00993320

\section{HAL Id: hal-00993320 \\ https://hal.science/hal-00993320}

Submitted on 20 May 2014

HAL is a multi-disciplinary open access archive for the deposit and dissemination of scientific research documents, whether they are published or not. The documents may come from teaching and research institutions in France or abroad, or from public or private research centers.
L'archive ouverte pluridisciplinaire HAL, est destinée au dépôt et à la diffusion de documents scientifiques de niveau recherche, publiés ou non, émanant des établissements d'enseignement et de recherche français ou étrangers, des laboratoires publics ou privés. 


\title{
Video Stream Transmodality
}

\author{
Pierre-Olivier Rocher ${ }^{12}$, Christophe Gravier ${ }^{1}$, Julien Subercaze ${ }^{12}$ and Marius Preda ${ }^{2}$ \\ ${ }^{1}$ Laboratoire Télécom Claude Chappe, télécom Saint-Etienne, Université Jean Monnet, F-42000 Saint-Etienne, France \\ ${ }^{2}$ Département ARTEMIS/GRIN, télécom SudParis, 91000 Evry, France \\ \{pierre-olivier.rocher, christophe.gravier, julien.subercaze\}@telecom-st-etienne.fr \\ marius.preda@telecom-sudparis.eu
}

\begin{abstract}
Keywords: $\quad$ video encoding; vectorization; modality;
Abstract: $\quad$ Transmodality is the partitioning of an image into regions that are expected to present a better entropy using different coding schemes, depending on their structural density, at constant bandwidth. In this paper we present the transmodality of video stream. Our contribution is a transmoder module that includes various different optimized video codecs and implements the concept of transmodality on a set of video streams. We evaluate our proposal with different kinds of video (in content term), and our algorithm shows comprehensive results by saving up to $8 \%$ of bandwidth for the same PSNR in comparison with the state-of-the-art video encoding baselines.
\end{abstract}

\section{INTRODUCTION}

Video communication accords for one of the highest development slope among various internet applications for the last few years. It is forecasted to be one of the main bandwidth consumer with respect to future applications. Different kinds of applications are using video compression: live TV, online newspapers, social networks... Over the last few years, new applications have emerged and one of the most important one is probably the Cloud Gaming. All cloud gaming applications like Gaikai ${ }^{1}$ or OnLive ${ }^{2}$ are based on remote rendering. Such a system is generally based on two parts. On the server side, the game is rendered and the current picture is encoded using a state-of-theart video encoder like MPEG-4 AVC (alias H.264), then streamed to the clients. Currently, for all previously described applications, the compression engine handles each image as an atomic element, regardless if the image contains more or less homogeneous regions. Adaptive encoding schemes bring new features, both in size and quality term, but the video is still processed as a pixel set. Some regions of the picture will be encoded with different parameters, but using the same global encoding scheme. The intuition behind this work is to go one step beyond by defin-

\footnotetext{
$1_{\text {http: //www.gaikai.com/ }}$

2 http://www.onlive.com/
}

ing an adaptive region-based encoding algorithm using fundamentally different encoding schemes. The encoding scheme should be chosen independently for each region, depending on its structure and heterogeneity. In this paper we propose several video coding systems to encode one video stream.

The remainder of this paper is organized as follows: Section 2 presents a review of background literature. Section 3 details the concept of transmodality. Section 4 presents our video encoding system from a global perspective to detailed components. Section 5 is dedicated to comparative testing and Section 6 concludes the paper and draws perspectives.

\section{RELATED WORK}

This section investigates existing work in the broad field of video encoding. While a complete state-ofthe-art on video coding is out of scope of this paper, we aim at focusing on relevant results in this part. We present two major encoding methods relevant to our approach: matrix-based and the one based on graphics primitives. We also present their applications to image and video coding. The initiated reader may skip this section. 


\subsection{Image}

We can consider two ways to store and manipulate a picture. The first one is a matrix-based representation, while the second one is based on graphics primitives. Nowadays, both solutions coexist but each representation brings specific advantages and disadvantages. The following paragraphs present respective benefits and drawbacks.

The matrix-based method is widely used for storing and sharing pictures. Such image representation, also known as raster or bitmap, is based on the pixel definition. Under this method, images are represented as 2D-matrices. This is a very convenient way for storing and even compressing pictures and pixelbased compression algorithms are proved to be quite efficient. The most common encoders are BMP, PNG, JPEG. Although, its major drawback is decrease quality while zooming into the parts of the picture. Even if the picture is not compressed, the original pixel element will eventually outsize the corresponding pixel in the screen space. At this point, the picture quality will strongly suffer from this limitation.

The second method does not use pixel as the basic element but instead makes use of graphic primitives. Each graphic primitive is a mathematical objet, like a point, a line or a polygon, and is defined by a formal definition. This representation then uses a set of graphic primitives to build an image. However, More complex objects like splines, Bezier curves or even non uniform rational basis spline (NURBS) can be used. Each graphic primitive is positioned in a reference system and bears specific attributes like its color, shape or thickness. Due to this approach of image representation, picture is first rendered and then displayed. i.e. every point in the reference system is computed using graphics primitives. The result of this computation is what is being displayed on the users device. The main advantage of this representation includes, independence from the rendered image size, its compacity and required data to reconstruct a vectorized picture cost the same price in size term.

When a picture or a video is live recorded, the raster format is used. Meantime, vectorization is the format of choice mainly for Computer-Aided Design (CAD) softwares. Due the increase of heterogeneity of display devices in our daily lives (IPTV, smartphones, tablets...) multimedia content is now often included in web pages. Whatever the screen's size, display picture quality is maximal.

Building a raster representation of a vector picture is a trivial task, but the reverse process is not obvious (Sun et al., 2007). References on this topic can be found (Lai et al., 2009), (Zhang et al., 2009), (Orzan et al., 2008), but proposed solutions are quite basic and are most often limited to black and white pictures processing. Some approaches to handle color exist but they cannot bear with photo realistic pictures (natural shots).

In proceeding sections, we distinguish codec types based on pixel and vector. The first one is related to a raster representation while the second one denotes the vector representation.

\subsection{Videos}

This subsection presents state-of-the-art video encoders (based on the MPEG-4 AVC video coding standard). Our main focus is on literature aiming to use several encoders to process a single video, but also on existing adaptive solutions. The first paragraph is a reminder on how a modern video encoder is working.

To compress a video, it is first splitted into a so called Group Of Pictures (GOP), which is basically made of three different types of pictures called $I, P$ and $B$. An I picture is a reference picture also known as a key frame. $\mathrm{P}$ pictures are predicted using a past referenced picture.

$\mathrm{B}$ pictures are bi-predicted, using both past and future $\mathrm{P}$ pictures (depending on the standard definition). Main steps of such encoders are prediction, transformation, quantization and entropy coding. In the encoder scheme the main step responsible of data compression is quantization. In order to control the final bitrate, the user can tune the quantization factor, called $Q p$ in most common implementations. Various strategies can be applied to control this parameter throughout the encoding process, like constant $Q p$ or constant bitrate (therefore with a non-constant $Q p$ ). A little $Q p$ value will ensure a good video quality. Furthermore usually, when $Q p=0$ the compression is lossless. Resulting entropy coded data, and necessary decoding information like prediction mode, motion vectors, quantization factor form the encoded video stream.

The previous paragraph described the main steps of a state-of-the-art video encoder. We now focus on systems using more than one video encoder to compress a video.

In (Chaudhury et al., 2011), (Khandelia et al., 2008) some macroblocks are encoded in a parametric manner, using an Auto Regressive (AR) process [9]. Selection of these macroblocks is done using a particular edge detection. Some moving textures can be modeled as a spatio-temporal AR process, which is in fact a tridimensional basis model version. In their system, I pictures are H.264 encoded, while P pic- 
tures are encoded using the proposed method. The process is based on $16 \times 16$ macroblock size (because of H.264). Macroblocks are then categorized into two sets: with or without edges, where macroblocks without edges are encoded with the proposed solution. The quality assessment was done using the Mean Opinion Score (MOS) system because the reconstruction scheme is statistically built. No information within the video like spatial resolution, frames per second or even related enconding time are exploited.

In (Zhu et al., 2007) authors offer to simply delete some macroblocks during the encoding process, in order to rebuild them by synthesis methods at the decoding stage. The method chosen to regenerate missing parts is the spatio-temporal patch searching. The system is running with an I-P-B picture scheme. Some tests have been conducted with QCIF videos, at 30 FPS using different $Q p$. Experimental results show a bitrate reduction of about $38.8 \%$ with a quite similar image quality (although no objective metrics were used).

In (Tripathi et al., 2012) the encoding process is based on both a traditional bloc coding and on a model coding. For such appraoch, they use a longterm temporal redundancy. Object detection related part is based on region of interest (ROI) algorithms, these ROIs are detected using principal component analysis (PCA). ROI areas are then segmented using graph-cut algorithms (Felzenszwalb and Huttenlocher, 2004). Finally a resultant area analysis is done, aiming to regroup some areas for whole optimization. A tracking algorithm is used for following ROIs in futures images. Theses ROIs areas, defined by a rectangle and an angle are finally coded using an active appearance model, which is statistically based.

While the literature provides several encoding systems using more than one encoder, these solutions apply only to $\mathrm{P}$ pictures. Our system aims at working on every picture of the video, and not only $\mathrm{P}$ pictures, like in (Chaudhury et al., 2011), (Khandelia et al., 2008).

Jointly to this research, work on adaptive video coding is largely present. One can discern two major trends: the first needs several versions of a same video; the second is based on a single version, which is adapted in realtime. The first solution is covered by the Scalable Video Coding (SVC), but also by the upcoming MPEG-DASH standard. Several recent articles can be found about the second adaptive way. In (Tizon et al., 2011a) real time adaptation is performed on video encoder as according to the network delay. Latency measurements are done before the launch of the system, but also periodically during the whole process. This latency evaluation makes possible the detection of network congestion and then the ability to take necessary actions to adapt the stream. In (Tizon et al., 2011a), they use a Cloud Gaming application, with an aim to to guarantee fluidity and responsiveness of the system. The proposed solution is to reduce the quantization parameter (among all macroblocks) and the necessary bitrate. In (Tizon et al., $2011 \mathrm{~b}$ ), the assumption between importants objects to the user and their position in the depth map is done. In other words, more the object is close to the user, more it is important. To reduce the necessary bitrate without loosing in quality, they refine the $Q p$ parameter, acting at macroblock level.

There is a common thread between all these solutions: they always use a set of pixel to encode and adapt the video coding. All presented solutions are using a standard version of H.264, We will use an optimized one. Adaptive technologies are largely used, but they are all the time confined to the pixel world. We will use an other type of encoding system, which is based on vectorization.

\section{TRANSMODALITY}

We introduce a new way of encoding a video stream using so called modalities. A modality is a set of areas to be compressed regarding a specific encoder. Each modality refers to (a) specific(s) part(s) in a frame, regarding an encoder especially well suited for encoding corresponding areas in a picture. According to our experiments we conclude that using more than one encoder will achieve a better compression rate than using only one, while preserving the same quality in terms of Peak Signal to Noise Ratio (PSNR). Conventional encoding approaches consider a video as a set of pixels and use a single encoding scheme for the whole set. Our approach is based on the assumption that a dynamic partitioning of the video frames and the approximation of some regions with a parametric representation will reduce the video bitrate. Splitting a video stream into objects and encoding them separatly is the basis of MPEG-4 part 2, but the way of doing this work on a video stream is not covered by this standard.

\subsection{Transmodality definition}

We define the Tansmodality as the fact of using several specific encoders to compress the same video file. The aim is to use a well-suited encoder to each region and using different modalities for video encoding. In 
other words, a modality is defined as being the compression of an area set by an encoder.

We define a class as a set of areas, each class being encoded using one specific encoder. Using one or more modalities is not known by advance but it belongs to the processed video itself. The decision to use 1, 2 or $n$ modalities is taken in real time during multimodal encoding, which means that shapes and area size are potentially different for each frame. For instance, Figure 1 is made of five areas $z_{1}$ to $z_{5}$ which are grouped into three classes $m_{1}$ to $m_{3}$. An area is a contiguous set of pixels, contiguity of two pixels is defined using the Kronecker operator (V4 neighborhood).

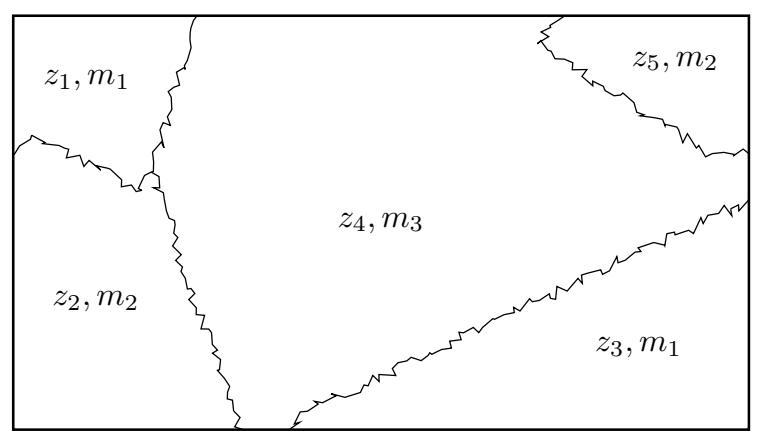

Figure 1: Areas and modalities in a sample picture.

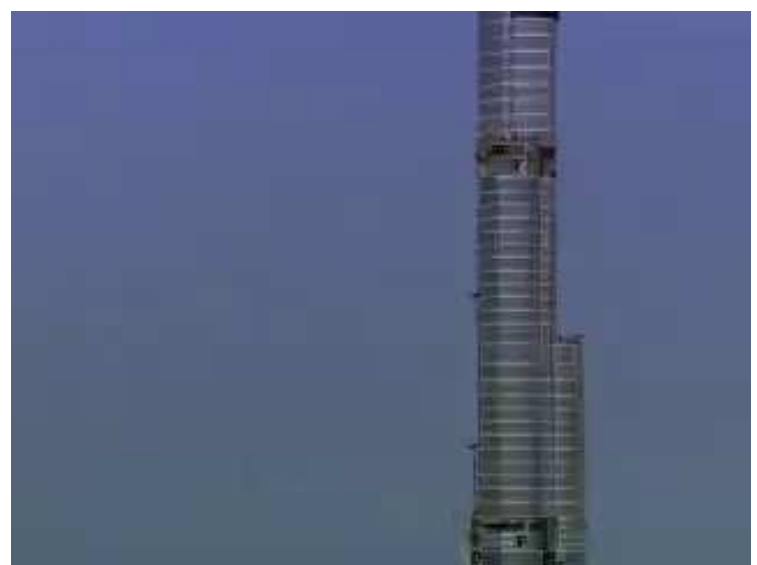

Figure 2: Input picture.

\subsection{Real example}

The whole splitting process is detailled step by step in upcoming parts. As a basic example, Figure 2 presents a sample picture extracted from the "Home" movie ${ }^{3}$. If we choose picture details (large amount of edges and colours) as a main criteria, then we can denote two kinds of modalities in this picture: a quite

\footnotetext{
${ }^{3}$ http: //home-2009.com/fr/
}

uniform, smooth one (i.e. the sky part), and another one which contains more details (the skyscrapper itself). Figure 3 outputs a possible splitting result. The reader can notice the rectangle at the bottom-left of the tower due to a macroblocking effect. This effect is discussed in Section 5.2.

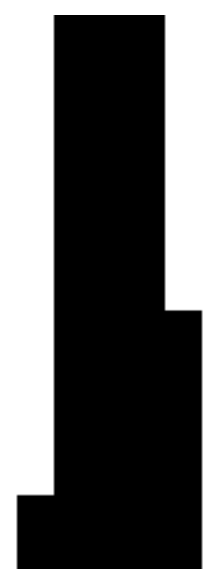

Figure 3: Level of detail based sample splitting.

The idea is to use several (two in this case) encoders to process a single video and to demonstrate that the corresponding multimodal video is smaller in size, while maintaining a good visual quality with minimal processing time. This approach raises several issues, including the choice of encoders and precision of the process to separate modalities or the way the output video stream will be written.

In the following section we focus on the transmoder whose role is to process the video compression with multi-modalities.

\subsection{Transmoder $v s$. Transcoder}

A transcoder is a software or hardware element which aims to modify the way how a video file or stream is compressed. It first decodes a video file or stream, and encodes it again using new parameters. This enables the application to modify the way this video will be transformed including three majors trends: the output video size (spatial resolution), the frame rate (temporal resolution) and the quality (resolution in PSNR). In this work, we introduce a new adjustable parameter called modality. Doing so, we define a new application called transmoder, which is a transcoder with an ability of using modalities, as described in previous sections. Our Transmoder as compared to transcoder uses an additional parameter that increase the output range of possible bitrates and thus make it possible to distribute the video to a larger number of people. A transmoder is also able to conduct simple transcod- 
ing operations, so with regard to our definition, the transcoder is a particular case of the transmoder.

In this work, we focus on implementing a transmoder that can handle two modalities, the pixel and the vector ones. What follows therefore presents our bimodal transmoder approach. In order to simplifiy, following parts of this article will rely on a transmoder working on two modalities. The multimodal process is then limited to a bimodal approach.

\section{Transmoder process}

Our transmoder is built on three separated parts: a decoder, an encoder and a sunder. The whole architecture is presented in Figure 4. The decoder decodes frames from a video file or stream. The sunder part is in charge of the partitioning of each frame into $n$ modalities. As depicted in Figure 4, the sunder output is a combination of $n$ modalities denoted M1 to $\mathrm{M} n$. Finally, the encoder uses the processed results of sunder as its input and encodes the whole video in a transmoded stream. The remaining systems blocks are explained in the following sections.

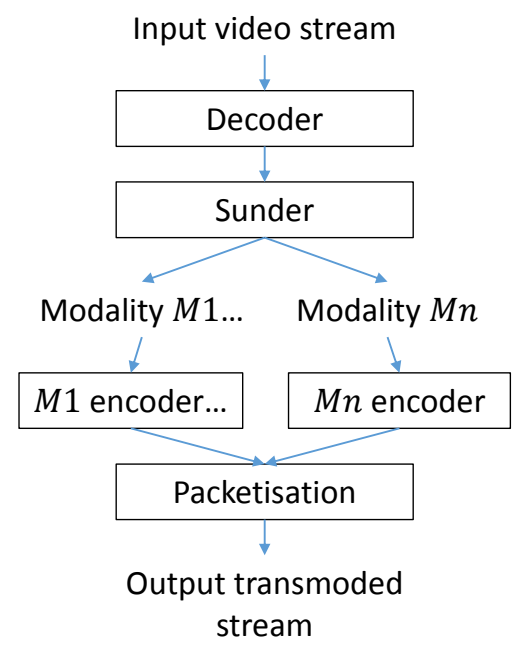

Figure 4: Synoptical scheme of the transmoding process.

\subsection{Decoding}

Like every transcoder, our transmoder has a decoding step. Decoding stage can be used or not either input data is in a compressed form or not. If the user wants to process a video file or stream, the decoding process provides a raw picture to the sunder using an appropriate decoder. An other solution is to catch a raw picture from a video game engine buffer for example and directly input it in the sunder.

\subsection{The Sunder}

The aim of Sunder is to take the decision of splitting (or not) the frame in several modalities. All the required step for such functionality as depicted in Figure 5 are explained later. But at this point we consider that the input of this whole process is an uncompressed picture, typically in an Red Green Blue (RGB) raw format. Some image processing treatments is first applied, then the picture is splitted into modalities (4.2.1). Once both modalities are splitted, respective encoding is conducted and some optimizations are applied on the pixel encoding part (4.2.2). The ouput of this process is a so-called bimodal picture. While all necessary informations are available, the video stream can be recorded. This is done by using packets, which are usually containing one frame. Packetisation process is described in (4.3.3).

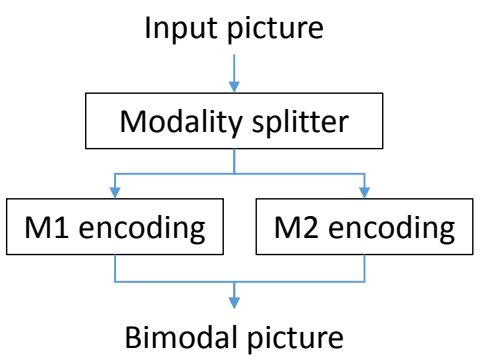

Figure 5: Synoptical scheme of the splitting process.

\subsubsection{Modality splitting}

Modality splitting involves with splitting operation by utilizing well known image processing filters. Previously, we explained our choice to split a picture into two modalities. To operate efficiently, an image processing filter appears to be the best choice. Our approach splits an image, based on the level of details which can be performed using an edge detector filter. There are various filters available for such requirements including Canny, Laplace an Sobel. For scalibility reasons, we require an algorithm that exhibits a good trade-off between performance an computation time. Under these conditions, (Heath et al., 1996) and more recenlty (Maini Raman, 2009) concludes that the Canny filter is the filter of choice. Furthermore, this choice of filter is affirmed by (Zhang et al., 2009) in which the authors processed videos. As an exemple, lets take the picture presented in Figure 2 as an input picture. After the edge detector process, we obtain a binary picture as displayed in Figure 6. As the reader can see, details in the picture are spotted in white, and more uniform areas are in black. Some 
morphological operations are now necessary to clean this mask (remove single points, close holes... ).

At this point, we need to make a choice regarding modality encoding. We choose to use one encoder from the pixel world and another one from the vector one. Chosen pixel encoder is the well-known MPEG4 AVC, the state-of-the-art reference. As literature does not provide any efficient library for a raster to vector convertion, thus we employ our own vectorization module. It is designed to suit our specific needs and is further described in 4.3.1. As we previously said, in the binary mask black parts represents a high amount of details while white parts depicts more uniform areas. Based on the characteristics of selected encoders, Black parts may be pixel coded whereas white parts may be vector coded. At this point, white areas are candidates to be vector coded, but further analysis (as described in 4.2.2) may downgrade them in the pixel world to ensure a good compacity in the output video stream. Because we choose to compress a modality using a MPEG-4 AVC encoder, the binary mask needs to be adapted since this kind of encoder is based on a macroblock definition (a $16 \times 16$ pixels size). This is simply done in checking each picture macroblocks: if all pixels are black, then the macroblock is black otherwise it is white. The corresponding new macroblock mask is depicted in Figure 7.

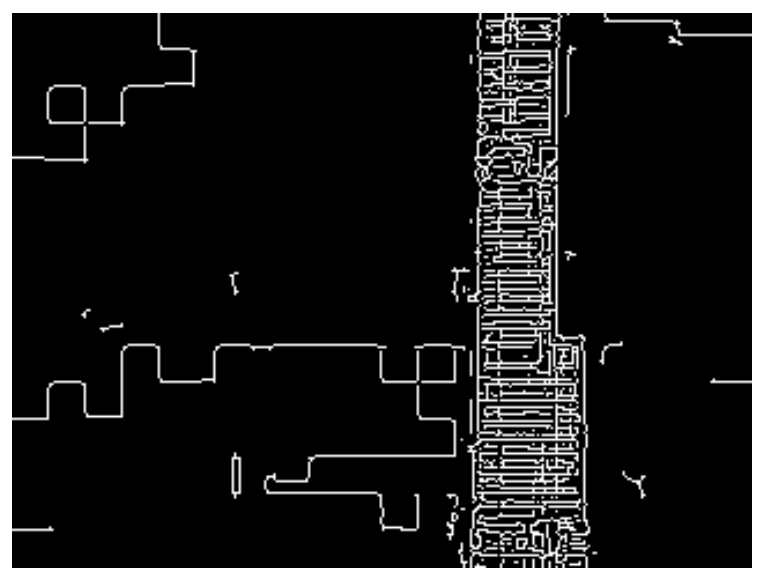

Figure 6: Canny filtered input picture representation.

\subsubsection{Analysis}

This concludes as the most important step of transmoding. All decisions made here are based on parameters set by the user at startup time. Currently a macroblock mask is present, the aim is now to process this mask and take appropriate decisions. Important steps of this analysis are presented in Figure 8.

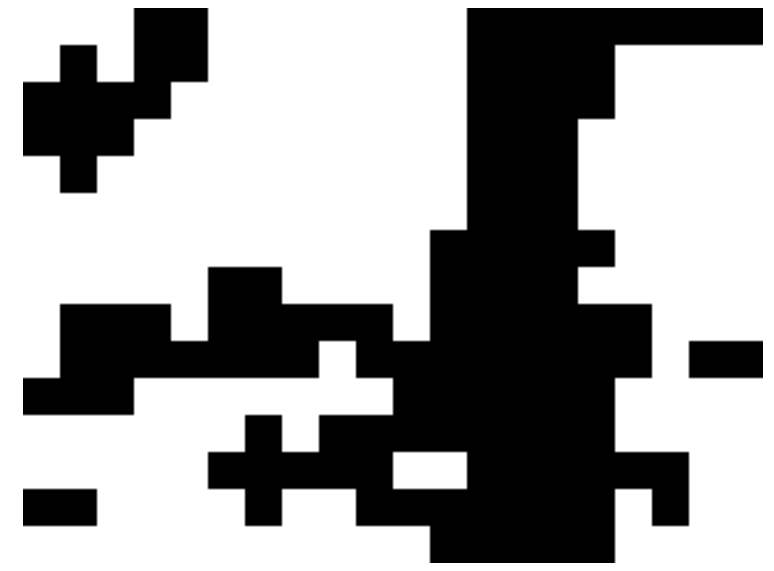

Figure 7: Macroblocked representation of vector areas (in white).

Labeling The first operation involves a V4 neighbourhood labelling of white areas. This will ensure that each vector area is separated from the others, and provides an internal data structure easy to manipulate.

First Filter Filter 1 as described in Figure 8 is responsible of the deletion of some area based on surface size criteria as according to the threshold set by the user. Consequently, some little areas which were planned to be vector encoded is in place pixel encoded, because in this case a pixel encoder offers better results. As an exemple, Figure 8 shows a possible output result.

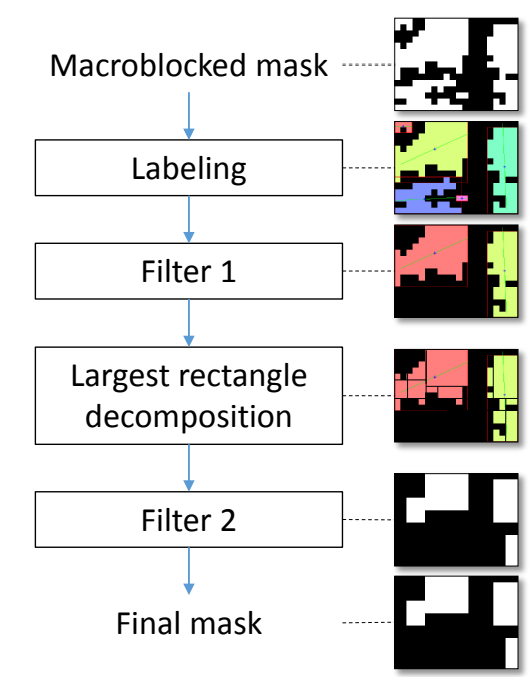

Figure 8: Logic analysis process in the sunder.

Largest rectangle decomposition According to Figure 8, we can now consider two areas. These areas can have any shape, including holes. To make the 
decoding stage possible, we will need to know two things: where are the vector areas and what are their respective definitions. We need now to consider how to store efficiently the binary mask. We can consider a lot of different solutions but in all cases, we need to keep a very good compacity. In order to fulfill this need, we choose to decompose any vector area into a set of largest rectangles. This kind of operation is known as a rectangle decomposition of binary matrix, henceforth DBMR problem. More information on this subject can be found in (Ferrari et al., 1984). Most of the time, taking into account the first two or three biggest rectangles is enough to keep a good approximation of the intial shape.

Second filter Our second filter aims at deleting little areas (user set thresold) that are moved in the pixel modality. At the end of this analysis, only important large rectangle areas are candidate for a parametrized representation. This process is detailed in Section 4.3.1.

This is the last step of sunder process. A binary mask specifies if each area has to be compressed using the first or the second modality. Thus encoding operations, related optimizations, and stream generation can be conducted.

\subsection{Stream encoding}

The modality splitting step is now finished. All pixel and vector areas are known and respective encoding operations can be conducted. The first paragraph describes our vectorization process while the second one is devoted to optimizations. Bimodal video stream is then packetized and dumped in an output video file.

\subsubsection{Vectorization}

As described earlier, vectorization is not a trivial task, especially when the user wants to process a natural picture and expect good quality results. The main problem of such encoding scheme includes complexity of such a project, necessary time to process pictures and output obtained sizes. All these parameters are intimately related to the picture content. The desirable objectives at this point is the implementation of specific software that meets our need along with a fast processing and a good quality (in term of PSNR). Because as the previous operations provides only a specific texture type and in order to achieve a uniform one, we can utilize a simple vectorization approach. As a first implementation we choose to simply use a polynomial based approach, inspired by what has been done in (Zhang et al., 2009). In order to vectorize an area, a 3D polynomial mean square based regression is computed using a matrix approach. Each polynomial expression is based on a static template of the form: $Z=a+b x+c y+d x^{2}+e x y+f y^{2}$. Where corresponding correlation coefficients are computed for each area. This determines the quality of the vectorization per area. If the result is too poor in quality, the corresponding area will fallback in the pixel mode.

\subsubsection{Encoding optimizations}

Encoding a single video using a per area optimized compression is not such a tedious task. The most difficult part is to use them correctly while avoiding redundancy in the data stream and trying to limit the impact on the encoding time. However, the only fact of adding some new lines of code will inevitably increase the necessary computing power.

Starting from this fact, we tried to minimize the impact of the sunder in optimizing the way encoder works. Both vector and pixel parts have been optimized. After all areas are vectorized, a process checks if some of them can be modeled using a same polynomial definition. Special treatment is reserved for writing vector data: for example, the number of digits is limited and all the data is compressed. The sunder process gives us some useful informations, which can be directly sent to the encoder. We can denote two optimizations: the first one (O1) directly indicates to the encoder macroblock to skip, and the second $(\mathrm{O} 2)$ manipulates the $Q p$ parameter. Each of these two optimization is macroblock based. The aim of $\mathrm{O} 1$ is to directly provide a list of macroblock to encoder that have to be skipped. Thus the analysis cost is reduced (minimize the encoding time) and the stream is more compact, regardless the chosen encoding preset. This optimization is only applied on P frames. O2 optimization is related to I and P frames. Per macroblock $Q p$ is modified to force the encoder to skip some macroblocks (thus which are not used by the pixel modality). These two optimizations can be used separately but also at the same time. They do not impact the quality of the output stream.

\subsubsection{Packetisation}

For being able to add our vectorization data part, we modify the way packetisation is done. Each vector data is just positionned after the picture data, hence a frame is a combination of pixel and vector data. Figure 9 presents a schematic representation of an H.264 stream on the left, and our modified one on the right. As the splitting is done live in an automatic manner, 

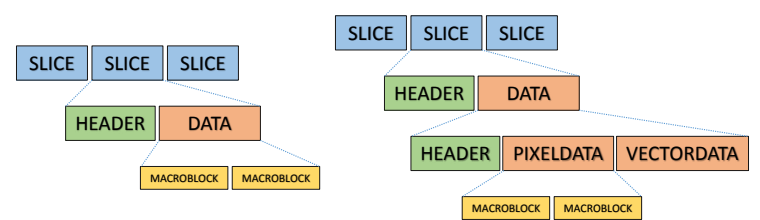

Figure 9: Usual H264 bitstream at left, our customized at right.

some packets may have only a pixel part, some others only a vector part. This stream is written in such a way that any compliant MPEG-4 AVC decoder will be able to read it, however only the pixel modality will be correctly displayed on the screen. In order to render and display the whole transmoded video stream, i.e. both pixel and vector modalities, a suitable decoder is necessary.

\section{Performance evaluation}

We evaluate our solution using videos from the real life. For such requirement, we download four different kinds of video from Youtube. We choose a documentary ${ }^{4}$, a music video clip ${ }^{5}$, a basketball movie ${ }^{6}$ and a video game screen record ${ }^{7}$. Content of such videos is made of slow and fast scenes, with any kind of areas: details, smooth parts... We see our transmoder as a standalone application. As a lot of parameters can be tuned and will inevitably lead to differents results, we choose to use static parameters for testing. Both modalities coding parameters are fixed to make comparisons possible. Furthermore the pixel video encoding strategy uses a constant quantization parameter, which is set by default to $Q p=20$. All testing movies have been resized to a $480 \mathrm{p}$ resolution, frame number varies from 300 to 500 pictures. Optimzations presented in 4.3.2 are used. Our transmoder implementation outputs XML files with all necessary informations, like frame sizes, made decisions... Results between a classical approach (transcoder) and our approach are then analysed.

\footnotetext{
${ }^{4}$ http: //www youtube. com/watch?v=jqxENMKaeCU, our shot start at 00:35:40 s

5 http: //www. youtube. com/watch?v= AgFeZr5ptV8\&list=TLQurwwErDm70

${ }^{6}$ http: / / www youtube. com/watch?v= 7 lvvoqEMHn 4 \& list=TLnMx 7 Qu I zwoM

${ }^{7}$ http: / / www youtube. com/watch?v=NC39Ww9ZLgs
}

Table 1: Obtained sizes in bytes after transcoding (TC) and transmoding (TM).

\begin{tabular}{|c|c|c|c|}
\hline Video name & TC size & TM size & \% diff \\
\hline Documentary & 32774899 & 30796069 & 6.04 \\
\hline Music clip & 2766735 & 2736886 & 1.08 \\
\hline Basketball & 4465946 & 4481855 & -0.35 \\
\hline Game screen rec. & 3105475 & 2838761 & 8.6 \\
\hline
\end{tabular}

\subsection{Implementation}

Video encoding is a quite heavy task even on most recent computers. The choice of a native programming language is then obvious. Because of code source portability but also external available libraries, the preferred choice for our implementation is $\mathrm{C} / \mathrm{C}++$. In order to accelerate the developping time and reduce the source code size to maintain, some well known multimedia libraries were used, such as FFmpeg and libx264 for decoding, encoding and manipulate the different kind of codec and containers. Image processing related operations are done by OpenCV, and our basic vectorization software uses the GNU Scientific library (GSL). The whole program is available as a standalone executable capable of transmoding any kind of videos, or as a library for direct splitting and encoding. Obviously the transmoder is also capable of simple transcoding operations. Although our model is designed for any number of modalities, our current implementation is limited to two: a pixel modality and a vector one.

All videos (Original, transcoded and transmoded versions) from the tests carried out in the results section are available ${ }^{8}$. Furthermore, necessary binary files are also available, thereby experiments can be reproduced by everyone.

\subsection{Results}

For our first experiment, we choose to compare the transcoded and transmoded size according the original file size. Both filters 1 and 2 were set to 1 , which means only one rectangle area is candidate for vectorization. Table 1 contains the result for all tested videos.

We can see that our transmoder provides better results in some cases, like with the documentary $6.04 \%$ better or with the game record $8.6 \%$ better. In some other cases, improvement is not significant (music clip video, $1.08 \%$ increase in efficiency), for some videos it may lead to a larger size. These observations

\footnotetext{
${ }^{8}$ http://datasets-satin.telecom-st-etienne. fr/porocher/videos/2013/
} 
Table 2: Quality assessement, using PSNR.

\begin{tabular}{|c|c|c|c|}
\hline Video name & O vs. TC & O vs. TM & TC vs. TM \\
\hline Documentary & 33.3664 & 33.7969 & 32.5094 \\
\hline Music clip & 40.0416 & 37.1115 & 36.2243 \\
\hline Basketball & 34.0789 & 35.9083 & 32.5832 \\
\hline Game screen rec. & 42.0799 & 43.0613 & 43.4582 \\
\hline
\end{tabular}

can be explained as our model is searching for smooth areas in order to provide a more compact representation. Ability of finding such areas is directly related to the video content, but also according video quality. If the video quality is poor, the macroblocking effect will provoke an over segmentation, resulting in less interesting results. Using a multiscale edge detector could solve this issue and will be investigated in further research.

\subsection{Quality assessement}

Quality assessment is not a trivial job and many well known metrics including PSNR or MSSIM are used to complete surveys. Our early tests use a PSNR metric for quality assessment, with some particularities. Indeed, we choose to compute a per modality and a per frame PSNR value, aiming to preserve the maximum amount of information for later analysis.

Results on the quality assessement are presented in Table 2. We denote three different tests. The original video ( $\mathrm{O}$ in the table) compared to the transcoded version (TC) and original video compared to the transmoded version (TM). Three times out of four, our transmoder gives better result compared to the transcoded version of the original video (Documentary, Basketball and Game screen record). For the documentary video, the PSNR increases, which means that the video quality is better, while necessary bitrate was reduced of about $6.04 \%$. For the game screen record: our transmoder saves $8.6 \%$ in bitrate while increasing the PSNR from 42.0799 to 43.0613.

As a short conclusion, we can say that our transmoder can provide better results both in bitrate and PSNR terms for most of the test videos.

\subsection{Real time processing}

Our first tests, presented in Table 3 reveal that real time processing is possible with videos assuming quite little spatial resolutions (240p). Using this spatial resolution, a 29 framerate is possible for the music clip. This framerate isn't reachable for all tested videos, as an example the documentary framerate is only about 7 pictures per second. In order to over-
Table 3: Transmoding time (second) vs. spatial resolution.

\begin{tabular}{|c|c|c|c|}
\hline Video name & $240 \mathrm{p}$ & $480 \mathrm{p}$ & $720 \mathrm{p}$ \\
\hline Documentary & 65.52 & 142.28 & 295.73 \\
\hline Music clip & 14.50 & 54.27 & 111.84 \\
\hline Basketball & 56.11 & 128.45 & 281.19 \\
\hline Game screen record & 18.03 & 113.08 & 261.17 \\
\hline
\end{tabular}

come this limitation, we first optimize the whole architecture of the software, especially by adding threading support in appropriate source code parts. Another way of increasing performance, especially in the image processing world is to use the GPU. This aspect has been brought to our program using OpenCV GPU related functions.

\section{Conclusion and future works}

In this paper, we presented a new kind of video encoding system called transmoder. The video stream is splitted into regions that are encoded using several modalities depending on the regions characteristics. We propose an overall system architecture for transmoding by employing two modalities, pixel and vector encoding. We first split each frame into region using an edge detector and then determine the more relevant encoder for each region. Where the resulted output of biomodal video stream is a combination of vector and pixel frames. We tested our approach against several real life videos and performance analysis shows that our approach significantly outperforms state-of-the-art encoder for a large majority of testbed videos. These tests present a reduction of the necessary bitrate up to $8 \%$. Future research will allow us to optimize even more our system, using for example GPU optimized algorithms. In the frame of our research on cloud gaming systems, we aim to integrate this multimodal coding scheme in the realtime rendering chain. We therefore adapt our approach for distributed processing in a cloud architecture. 


\section{REFERENCES}

Chaudhury, S., Mathur, M., Khandelia, A., Tripathi, S., Lall, B., Roy, S. D., and Gorecha, S. (2011). System and method for object based parametric video coding. U.S. Classification: 375/240.16; 375/240.25; 375/E07.027; 375/E07.076

Felzenszwalb, P. F. and Huttenlocher, D. P. (2004). Efficient graph-based image segmentation. International Journal of Computer Vision, 59(2):167-181.

Ferrari, L., Sankar, P., and Sklansky, J. (1984). Minimal rectangular partitions of digitized blobs. Computer Vision, Graphics, and Image Processing, 28(1):58-71.

Heath, M., Sarkar, S., Sanocki, T., and Bowyer, K. (1996). Comparison of edge detectors: a methodology and initial study. In Proceedings CVPR '96, 1996 IEEE Computer Society Conference on Computer Vision and Pattern Recognition, 1996, pages 143-148.

Khandelia, A., Gorecha, S., Lall, B., Chaudhury, S., and Mathur, M. (2008). Parametric video compression scheme using AR based texture synthesis. In Sixth Indian Conference on Computer Vision, Graphics Image Processing, 2008. ICVGIP '08, pages 219-225.

Lai, Y.-K., Hu, S.-M., and Martin, R. R. (2009). Automatic and topology-preserving gradient mesh generation for image vectorization. ACM Trans. Graph., 28(3):85:185:8.

Maini Raman, D. H. A. (2009). Study and comparison of various image edge detection techniques. International Journal of Image Processing.

Orzan, A., Bousseau, A., Winnemller, H., Barla, P., Thollot, J., and Salesin, D. (2008). Diffusion curves: a vector representation for smooth-shaded images. ACM Trans. Graph., 27(3):92:192:8.

Sun, J., Liang, L., Wen, F., and Shum, H.-Y. (2007). Image vectorization using optimized gradient meshes. In ACM SIGGRAPH 2007 papers, SIGGRAPH '07, New York, NY, USA. ACM.

Tizon, N., Moreno, C., Cernea, M., and Preda, M. (2011a). MPEG-4-based adaptive remote rendering for video games. In Proceedings of the 16th International Conference on 3D Web Technology, Web3D '11, pages 45-50, New York, NY, USA. ACM.

Tizon, N., Moreno, C., and Preda, M. (2011b). ROI based video streaming for $3 \mathrm{D}$ remote rendering. In 2011 IEEE 13th International Workshop on Multimedia Signal Processing (MMSP), pages 1-6.

Tripathi, S., Mathur, M., Dutta Roy, S., and Chaudhury, S. (2012). Region-of interest based parametric video compression scheme. Communicated to the IEEE Transactions on Circuits and Systems for Video Technology.

Zhang, S.-H., Chen, T., Zhang, Y.-F., Hu, S.-m., and Martin, R. (2009). Vectorizing cartoon animations. IEEE Transactions on Visualization and Computer Graphics, 15(4):618 -629.
Zhu, C., Sun, X., Wu, F., and Li, H. (2007). Video coding with spatio-temporal texture synthesis. In ICME, pages $112-115$. 